\title{
Influência da radiação ultravioleta e aditivos na conservação de kiwis minimamente processados
}

\author{
Influence of ultraviolet radiation and additives on minimally \\ processed kiwi conservation
}

Caroline Farias Barreto $^{1}$ (D), Renan Navroski ${ }^{1 *}$ (D), Léo Omar Duarte Marques ${ }^{1}$ (D), Rodrigo Fernandes dos Santos ${ }^{1}$ (D), Marcelo Barbosa Malgarim ${ }^{1}$ (D) , Carlos Roberto Martins ${ }^{2}$

${ }^{1}$ Universidade Federal de Pelotas (UFPel), Faculdade de Agronomia Eliseu Maciel, Programa de Pós-graduação em Agronomia, Capão do Leão/RS - Brasil

${ }^{2}$ Empresa Brasileira de Pesquisa Agropecuária (Embrapa Clima Temperado), Pelotas/RS - Brasil

${ }^{*}$ Corresponding Author: Renan Navroski, Universidade Federal de Pelotas (UFPel), Faculdade de Agronomia Eliseu Maciel, Programa de Pós-graduação em Agronomia, Campus UFPel, Av. Eliseu Maciel, s/n, Caixa Postal: 354, CEP: 96160-000, Capão do Leão/RS - Brasil, e-mail: navroski@outlook.com

Cite as: Barreto, C. F., Navroski, R., Marques, L. O. D., Santos, R. F., Malgarim, M. B., \& Martins, C. R. (2021) Influence of ultraviolet radiation and additives on minimally processed kiwi conservation. Brazilian Journal of Food Technology, 24, e2020024. https://doi.org/10.1590/1981-6723.02420

\begin{abstract}
Resumo
Kiwis minimamente processados necessitam de técnicas para manter a qualidade dos frutos durante a conservação e o período de prateleira. Aditivos combinados à radiação ultravioleta podem ser uma alternativa para assegurar a qualidade das frutas por mais tempo de conservação. Neste contexto, foi proposto avaliar a eficiência do uso da radiação UV-C e diferentes aditivos na prevenção do escurecimento e conservação de kiwis 'Bruno' minimamente processados. As fatias de kiwi foram submetidas a soluções contendo os seguintes tratamentos: controle, ácido ascórbico a $1 \%$; isoascorbato de sódio a 1\%; ácido cítrico a 1\%, depois, utilizou-se por dois minutos a radiação UV$\mathrm{C}$ na intensidade de $2,71 \mathrm{~kJ} \mathrm{~m}^{-2}$. As bandejas foram armazenadas em câmara fria a $4 \pm 1{ }^{\circ} \mathrm{C}$ de temperatura, com umidade relativa de $85 \%$ a $90 \%$, e armazenadas por períodos de 3, 6 e 9 dias. Avaliaram-se perda de massa, coloração da polpa, sólidos solúveis, acidez titulável, incidência de podridão, compostos fenólicos totais e atividade antioxidante. $\mathrm{O}$ uso de aditivos melhora a conservação do kiwi minimamente processado, com destaque para o isoascorbato de sódio e o ácido cítrico. Os frutos de kiwi da cultivar 'Bruno' podem ser armazenados por até 6 dias sob refrigeração com o uso de aditivos. Após esse período, a perda de massa é elevada. O uso de UV-C não alterou a qualidade dos kiwis durante o armazenamento, podendo ter seu uso dispensado.
\end{abstract}

Palavras-chave: Actinidia deliciosa; Armazenamento; UV-C; Isoascorbato; Acído cítrico; Qualidade.

\begin{abstract}
Minimally processed kiwis need techniques to maintain the quality of the fruits during conservation and shelf life. The additives combined with ultraviolet radiation can be an alternative to ensure the quality of the fruits for a longer conservation period. In this context, it was proposed to evaluate the efficiency of the use of UV-C radiation and different additives in preventing the browning and conservation of minimally processed 'Bruno' kiwis. The kiwi slices
\end{abstract}


were subjected to solutions containing the following treatments: control, ascorbic acid $1 \%$; $1 \%$ sodium isoascorbate; citric acid 1\%, after two minutes UV-C radiation was used at an intensity of $2.71 \mathrm{~kJ} \mathrm{~m}^{-2}$. The trays were stored in a cold chamber at $4 \pm 1^{\circ} \mathrm{C}$ of temperature, with a relative humidity of $85 \%$ to $90 \%$, and stored for periods of 3,6 and 9 days. It was evaluated: mass loss, pulp color, soluble solids, titratable acidity, the incidence of rot, total phenolic compounds, and antioxidant activity. The use of additives improves the conservation of minimally processed kiwi, with emphasis on sodium isoascorbate and citric acid. The kiwi fruits of the cultivar 'Bruno' can be stored for up to 6 days under refrigeration with the use of additives, after which the loss of mass is high. The use of UV-C did not change the quality of kiwis during storage, and their use may be dispensed with.

Keywords: Actinidia deliciosa; Storage; UV-C; Isoascorbate; Citric acid; Quality.

\section{Introdução}

A maior parte do kiwi consumido no Brasil é proveniente de importações. Em 2016, o Brasil importou aproximadamente 30 mil toneladas de kiwi (Food and Agriculture Organization, 2018), demonstrando potencial na expansão da cultura, ao mesmo tempo que constitui um desafio, considerando-se a deficiência de tecnologias de manejo durante a produção e após a colheita dos frutos (Pegoraro et al., 2016). A vida útil do kiwi é extremamente dependente do adequado manejo pós-colheita. Essa fruta apresenta alta sensibilidade ao etileno, causando amolecimento rápido da polpa, além de suscetibilidade à ação de fungos que infectam os frutos (Blum \& Ayub, 2009), características que comprometem a comercialização.

Os consumidores de frutas estão cada vez mais preocupados em consumir alimentos benéficos à saúde. Sendo assim, tem aumentado a preferência por frutos frescos (Marques et al., 2011). Alimentos minimamente processados estão associados à qualidade de frutas e hortaliças "frescas" (Rodgers, 2016). Atualmente, frutas e legumes minimamente processados são particularmente apreciados pelos consumidores, devido às suas propriedades nutritivas, conveniência e facilidade de consumo (Giovenzana et al., 2015). Considerando o apelo nutricional e o atendimento a uma demanda crescente por produtos prontos para consumo, empresas vêm se especializando no processamento mínimo de frutas e hortaliças, preocupadas também com a qualidade microbiológica (Maldonade et al., 2019). Nesse contexto, o processamento mínimo se insere e torna-se uma forma de aproveitar excedentes de produção e agregar valor ao produto (Vilas-Boas et al., 2009; Marques et al., 2011), sendo primordial assegurar a segurança microbiológica e a qualidade do alimento (Maldonade et al., 2019).

Para evitar perdas de coloração e escurecimento e manter a qualidade nutricional dos produtos minimantes processados, têm sido utilizado aditivos, como ácidos ascórbico (Kluge et al., 2014; Ambuko et al., 2017; Mendoza-Goméz et al., 2017) e cítrico (Kluge et al., 2014) e isoascorbato de sódio (Martins et al., 2011; Barreto et al., 2016; Mendoza-Goméz et al., 2017). O ácido cítrico atua na inibição de óxidos de polifenóis, visto que o escurecimento das frutas ocorre quando há altos teores de fenóis e ação da enzima polifenol oxidase (PPO), que provoca colorações de tonalidade marrom nos frutos e alterações no sabor (Manolopoulou \& Varzakas, 2011). O uso de isoascorbato de sódio auxilia na manutenção da coloração dos frutos minimantes processados, ou seja, retarda o escurecimento da polpa (Barreto et al., 2016; MendozaGoméz et al., 2017), pois é capaz de diminuir a atividade de enzimas responsáveis pela deterioração de frutos, como a PPO, que provoca escurecimento (Mendoza-Goméz et al., 2017). O ácido ascórbico é capaz de manter boa sanidade microbiana nos alimentos, mantendo as características sensoriais originais (MendozaGoméz et al., 2017).

Além da aplicação dos aditivos, pode-se associar o uso de radiação utravioleta (UV-C) nos produtos minimantes processados (Vieites et al., 2004; Manzocco et al., 2011; Barreto et al., 2016; Chen et al., 2016). Baixas doses de radiação ultravioleta (UV-C) possuem potenciais como tratamento pós-colheita, com o intuito de sanitizar e conservar os produtos minimantes processados (Manzocco et al., 2011; Maharaj, 2015). Em um estudo com maçãs minimamente processadas, o uso de aditivos e UV-C reduziu a incidência de 
podridões, além de aumentar o teor de sólidos solúveis, fenóis totais e atividade antioxidante após 12 dias de armazenamento (Barreto et al., 2016).

A tecnologia do uso de UV-C apresenta como vantagem a inativação de microrganismos patogênicos e deteriorantes, com mínima perda da qualidade nutricional, sem gerar resíduo (Ochoa-Velasco \& GuerreroBeltrán, 2012; Manzocco et al., 2011; Gayán et al., 2014). Entretanto, a exposição a altas doses de UV-C pode danificar e enfraquecer os tecidos, favorecendo a proliferação de possíveis patógenos (Fan et al., 2017).

Um dos principais desafios do processamento mínimo é potencializar o período de conservação e preservar a qualidade de frutas minimamente processadas (Wu et al., 2012). O kiwi possui alto teor de umidade, sendo um produto altamente perecível (Chin et al., 2015). Portanto, é desafiador manter o produto fresco, pois perde gradualmente o frescor após alguns dias (Chakraborty et al., 2020). Segundo Heiffig et al. (2006), os kiwis minimante processados possuem vida de prateleira entre seis e nove dias. Deste modo, são necessários estudos para manter a qualidade e prolongar o período de comercialização de kiwis minimamente processados.

Embora o kiwi seja amplamente consumido no Brasil, até o momento existem poucos dados sobre a conservação desse fruto quando minimamente processado. Deste modo, um estudo propôs-se avaliar a combinação da radiação UV-C com diferentes aditivos em relação à qualidade e à conservação de kiwis minimamente processados.

\section{Material e métodos}

Os kiwis da cultivar 'Bruno' utilizados no experimento foram produzidos em pomar comercial no município de Pelotas, no Rio Grande do Sul, Brasil. As frutas utilizadas foram colhidas no ciclo de 2017.

O processamento mínimo foi conduzido no Laboratório de Agronomia da Universidade Federal de Pelotas. As frutas foram pré-selecionados de acordo com a ausência de injúrias e incidência visuais de doenças. Após a seleção dos frutos, estes foram sanitizados com hipoclorito de sódio a $200 \mathrm{mg} \mathrm{L}^{-1}$, por dez minutos, em temperatura ambiente. Os kiwis selecionados foram cortados em quatro fatias com o auxílio de facas de aço inox.

O delineamento experimental utilizado foi inteiramente casualizado, com esquema fatorial $4 \times 2 \times 4$ (quatro aditivos $\times$ duas aplicações de UV-C $\times$ quatro períodos de armazenamento), com quatro repetições, sendo cada uma composta de nove fatias de kiwi minimamente processadas. As fatias de kiwi foram imersas por dois minutos nas soluções contendo os seguintes tratamentos: controle (água destilada); ácido ascórbico (AA) a 1\% (m/v) (Moreno et al., 2016); isoascorbato de sódio (IS) a 1\% (m/v) (Barreto et al., 2016); ácido cítrico (AC) a 1\% (m/v) (Martins et al., 2011). Em seguida, os pedaços foram drenados, por três minutos, para eliminar o excesso de solução, e as fatias foram secas em ambiente refrigerado $\left(10 \pm 1{ }^{\circ} \mathrm{C}\right)$, por dez minutos.

Os pedaços de kiwi foram colocados em bandejas de poliestireno expandido de $300 \times 230 \times 33$ milímetros, tendo sido embaladas com filme PVC esticável de $9 \mu$. Utilizou-se radiação UV-C "Phillips ${ }^{\circledR}$ " $30 \mathrm{~W}$ nos kiwis minimamente processados. A distância entre as lâmpadas e as bandejas foi de, aproximadamente, 50 centímetros e a intensidade da radiação emitida pelas lâmpadas foi quantificada com um medidor de luz UV digital (RS-232 Modelo MRUR-203, "Instrutherm"), resultando em intensidade de 2,71 kJ m por aplicação em dois minutos. Em seguida, as bandejas foram armazenadas em câmara fria a $4 \pm 1{ }^{\circ} \mathrm{C}$ de temperatura, sob umidade relativa de $85-90 \%$, tendo sido armazenadas pelos períodos de $0,3,6$ e 9 dias.

Para monitorar a conservação dos kiwis durante o armazenamento, avaliaram-se as seguintes variáveis:

- Perda de massa fresca, considerando-se a diferença entre a massa inicial dos kiwis minimamente processados e aquela obtida ao final de cada tempo de armazenamento, de acordo com a fórmula: perda de massa $=[($ massa inicial - massa final $) /($ massa inicial $)] \times 100$, e os resultados foram expressos em porcentagem de perda de massa $(\%)$; 
- Coloração da polpa, medida com colorímetro (Minolta CR-300 ${ }^{\circledR}$, Japão) com fonte de luz D65, sendo expressa pelo sistema de coordenadas retangulares $\mathrm{L}^{*}, \mathrm{a}^{*}, \mathrm{~b}^{*}$, conforme a CIE (Comission Internatinale de E'clairage), onde $L^{*}$ é expressa em valores de luminosidade, $a^{*}$ representa a cor vermelha $(+)$ ou verde $(-), b^{*}$, a cor amarela (+) ou azul (-) e a matiz ou tonalidade cromática representada pelo ${ }^{\circ}$ Hue e Croma, tendo sido realizadas duas leituras em cada fatia;

- Sólidos solúveis foram obtidos pelo refratômetro digital (Atago ${ }^{\circledR}$, Brasil), expresso em ${ }^{\circ}$ Brix do suco;

- Acidez titulável com $10 \mathrm{~mL}$ de suco diluídos em $90 \mathrm{~mL}$ de água destilada e titulados até pH 8,1 com solução de $\mathrm{NaOH}$ 0,1. Os resultados foram expressos em porcentagem de ácido cítrico (Instituto Adolfo Lutz, 2008);

- Compostos fenólicos totais determinados pelo método baseado na reação com o reagente Folin-Ciocalteu, conforme o método adaptado de Singleton et al. (1999), tendo os resultados sido expressos em miligramas de equivalentes de ácido gálico (EAG) por grama de amostra fresca mg EAG $100 \mathrm{~g}^{-1}$;

- Atividade antioxidante determinada pelo método do radical DPPH adaptado de Brand-Williams et al. (1995), com os resultados expressos em mg equivalente de Trolox $100 \mathrm{~g}^{-1}$ de fruto.

Os dados obtidos foram submetidos à análise de variância $(p \leq 0,05)$. Ao ser constatada significância estatística, procedeu-se à análise entre as médias pelo teste de Tukey $(p<0,05)$ para comparar os tratamentos.

\section{Resultados e discussão}

Os quadrados médios da análise de variância dos atributos avaliados evidenciam que a acidez titulável e a perda de massa apresentaram interação entre os três fatores estudados (Tabela 1). O teor de sólidos solúveis apresentou interação apenas entre os períodos de armazenamento e os aditivos aplicados. Com fenóis e matriz do ângulo Hue, houve interação entre a aplicação de UV-C e os períodos, enquanto atividade antioxidante, luminosidade e cromaticidade não apresentaram interação entre os fatores testados.

Menor perda de massa aos nove dias de armazenamentos foi observada nos kiwis tratados com IS e sem UV-C em relação ao controle sem UV-C e IS com UV-C (Tabela 2). Em maçãs 'Fuji' minimamente processadas a perda de massa foi menor no tratamento controle sem radiação UV-C em relação ao uso de radiação UV-C (Barreto et al., 2016). Por outro lado, Chen et al. (2016) observaram que o uso de UV-C associado ao AC contribuiu para reduzir a perda de massa em maçãs 'Fuji' minimamente processadas. A exposição prolongada à radiação U-VC pode desintegrar membranas celulares e favorecer desidratação e consequente aumento da perda de massa (Manzocco et al., 2011; Chen et al., 2016). Segundo esses mesmos autores, a radiação pode reduzir a perda de massa somente se aplicada em intensidade moderada.

A perda de massa dos kiwis aos 9 dias de armazenamento foi de $12 \%$ e $9,34 \%$ para o tratamento controle (sem o uso de UV-C e aditivos) e IS com UV-C, respectivamente (Tabela 2). Carvalho \& Lima (2002) encontraram perdas de massa próxima a $1 \%$ em kiwis minimamente processados ao longo de 10 dias de armazenamento refrigerado, Agar et al. (1999), perdas próximas a 0,6\% e Heiffig et al. (2006) observaram perdas de massa de $1,2 \%$ aos 12 dias de armazenamento.

Observa-se que o uso de UV-C ou não no terceiro e no sexto dia de armazenamento refrigerado não interferem na acidez titulável dos kiwis minimamente processados (Tabela 2). Somente aos nove dias de armazenamento houve diferenças entre o uso de radiação UV-C e aditivos. A acidez titulável manteve alta com o uso de UV-C combinado com a aplicação de AA aos nove dias de armazenamento refrigerado, diferindo do controle sem aplicação de UV-C no mesmo período (Tabela 2). Em frutos de kiwi, a acidez titulável geralmente diminui após os dias de armazenamentos (Pal et al., 2015). Considerando essa afirmação, o único tratamento que não apresentou variação de acidez titulável ao longo dos períodos de armazenamento foi a combinação de aplicação de AC e UV-C. 
Tabela 1. Quadrados médios de atributos físico-químicos de frutos de kiwi armazenados sob refrigeração e submetidos a tratamento com UV-C e aditivos

\begin{tabular}{|c|c|c|c|c|c|c|c|c|c|c|c|c|c|c|c|c|c|c|c|c|c|}
\hline Parâmetro & GL & P. Massa & & AT & & Firmeza & & pH & & Fenóis & & Hue & & SS & & Luminosidade & & Atividade antioxidante & & Croma & \\
\hline $\mathrm{UV}-\mathrm{C}$ & 1 & 0,790 & ns & 0,0001 & $*$ & 8,862 & $*$ & 0,009 & ns & 23,146 & ns & 0,147 & ns & 3,099 & $*$ & 24,160 & ns & 38926,407 & $\mathrm{~ns}$ & 0,108 & ns \\
\hline Aditivos & 3 & 22,209 & $* *$ & 1,121 & ns & 15,892 & $* *$ & 0,006 & ns & 245,573 & ns & 6,520 & $* *$ & 6,656 & $* *$ & 28,843 & $* *$ & 77412,469 & $* *$ & 6,926 & ns \\
\hline Períodos & 3 & 92,604 & $* *$ & 26,630 & $* *$ & 65,935 & $* *$ & 0,737 & $* *$ & 7549,747 & $* *$ & 94,686 & $* *$ & 6,701 & $* *$ & 477,632 & $* *$ & 35804,026 & $* *$ & 638,827 & $* *$ \\
\hline $\mathrm{U} \times \mathrm{A}$ & 3 & 21,050 & $* *$ & 6,918 & $* *$ & 7,474 & $* *$ & 0,006 & ns & 201,968 & ns & 0,950 & ns & 0,671 & $\mathrm{~ns}$ & 9,346 & ns & 19819,253 & ns & 7,324 & $\mathrm{~ns}$ \\
\hline $\mathrm{U} \times \mathrm{P}$ & 3 & 0,541 & $\mathrm{~ns}$ & 2,414 & $* *$ & 11,271 & $* *$ & 0,020 & $* *$ & 268,903 & $*$ & 4,887 & $* *$ & 0,458 & $\mathrm{~ns}$ & 12,134 & ns & 28379,207 & $\mathrm{~ns}$ & 4,091 & ns \\
\hline $\mathrm{P} \times \mathrm{A}$ & 9 & 10,588 & $* *$ & 0,810 & ns & 3,558 & $*$ & 0,015 & $* *$ & 99,992 & $\mathrm{~ns}$ & 1,686 & $\mathrm{~ns}$ & 1,673 & $*$ & 3,989 & ns & 15757,914 & ns & 2,627 & $\mathrm{~ns}$ \\
\hline $\mathrm{U} \times \mathrm{A} \times \mathrm{P}$ & 9 & 15,905 & $* *$ & 4,393 & $* *$ & 1,700 & $\mathrm{~ns}$ & 0,005 & ns & 170,746 & ns & 1,301 & ns & 0,672 & $\mathrm{~ns}$ & 8,611 & ns & 15027,210 & ns & 3,936 & ns \\
\hline Tratamentos & 31 & 20,918 & $* *$ & 5,099 & $* *$ & 11,545 & $* *$ & 0,080 & $* *$ & 879,302 & $* *$ & 11,231 & $* *$ & 2,183 & $* *$ & 55,529 & $\mathrm{~ns}$ & 56998,432 & $* *$ & 65,505 & $* *$ \\
\hline Resíduo & 64 & 1,704 & & 0,579 & & 1,694 & & 0,003 & & 89,858 & & 0,839 & & 0,742 & & 6,506 & & 15601,599 & & 5,275 & \\
\hline
\end{tabular}

**Significativo ao nível de $1 \%$ de probabilidade $(p<0,01)$ * Significativo ao nível de $5 \%$ de probabilidade $(0,01 \leq p<0,05)$. ns: não significativo $(p \geq 0,05)$. GL $=$ graus de liberdade; AT $=$ acidez titulável; $\mathrm{SS}=$ sólidos solúveis. 
Tabela 2. Perda de massa e acidez titulável em frutos de kiwi minimamente processados com $0,3,6$ e 9 dias de armazenamento.

\begin{tabular}{|c|c|c|c|c|c|}
\hline & & Dia 0 & Dia 3 & Dia 6 & Dia 9 \\
\hline \multicolumn{6}{|c|}{ Perda de massa } \\
\hline \multirow{4}{*}{$\begin{array}{l}\text { Com } \\
\text { UV-C }\end{array}$} & Controle & $0,0^{\mathrm{aA}}$ & $0,84 \pm 0,84 \mathrm{aB}$ & $2,09 \pm 1,81 \mathrm{aAB}$ & $4,44 \pm 2,25 \mathrm{bA}$ \\
\hline & $\mathrm{AA}$ & $0,0 \mathrm{aB}$ & $1,67 \pm 1,15$ aA & $0,94 \pm 0,05$ aA & $2,64 \pm 0,51 \mathrm{bcA}$ \\
\hline & IS & $0,0^{\mathrm{aA}}$ & $0,92 \pm 0,14 \mathrm{aB}$ & $0,97 \pm 0,09 \mathrm{aB}$ & $9,34 \pm 4,36^{\mathrm{aA}}$ \\
\hline & $\mathrm{AC}$ & $0,0^{\mathrm{aC}}$ & $0,56 \pm 0,49$ aA & $0,97 \pm 0,99$ aA & $2,38 \pm 0,56^{\text {bcA }}$ \\
\hline \multirow{4}{*}{$\begin{array}{c}\text { Sem } \\
\text { UV-C }\end{array}$} & Controle & $0,0^{\mathrm{aA}}$ & $0,96 \pm 0,08^{\mathrm{aBC}}$ & $3,48 \pm 2,12 \mathrm{aB}$ & $12,07 \pm 3,96^{\mathrm{aA}}$ \\
\hline & $\mathrm{AA}$ & $0,0^{\mathrm{aA}}$ & $0,32 \pm 0,56^{\mathrm{aA}}$ & $0,31 \pm 0,54$ aA & $1,65 \pm 0,61 \mathrm{bcA}$ \\
\hline & IS & 0,0 aA & $0,69 \pm 0,60$ aA & $1,06 \pm 0,11$ aA & $0,66 \pm 0,58^{\mathrm{cA}}$ \\
\hline & $\mathrm{AC}$ & $0,0^{\mathrm{aA}}$ & $0,34 \pm 0,58$ aA & $0,71 \pm 0,62$ aA & $2,63 \pm 0,87 \mathrm{bcA}$ \\
\hline \multicolumn{6}{|c|}{ Acidez titulável } \\
\hline \multirow{4}{*}{$\begin{array}{c}\text { Com } \\
\text { UV-C }\end{array}$} & Controle & $19,53 \pm 1,10^{\mathrm{aA}}$ & $16,00 \pm 0,26^{\mathrm{aB}}$ & $15,67 \pm 0,31 \mathrm{aB}$ & $16,43 \pm 0,40$ abB \\
\hline & $\mathrm{AA}$ & $19,60 \pm 0,26^{\mathrm{aA}}$ & $15,93 \pm 0,25 \mathrm{aB}$ & $15,50 \pm 1,28^{a B}$ & $17,10 \pm 0,87 \mathrm{aB}$ \\
\hline & IS & $17,23 \pm 1,45$ bcA & $15,97 \pm 0,51^{\mathrm{aAB}}$ & $16,30 \pm 0,75^{\mathrm{aAB}}$ & $15,23 \pm 0,25 \mathrm{abcB}$ \\
\hline & $\mathrm{AC}$ & $15,97 \pm 0,64 \mathrm{cA}$ & $15,10 \pm 0,61^{\mathrm{aA}}$ & $16,50 \pm 1,15$ aA & $15,17 \pm 1,29$ abcA \\
\hline \multirow{4}{*}{$\begin{array}{c}\text { Sem } \\
\text { UV-C }\end{array}$} & Controle & $16,07 \pm 0,99 \mathrm{cA}$ & $15,57 \pm 1,08^{\mathrm{aAB}}$ & $17,20 \pm 0,53$ aA & $14,27 \pm 0,15 \mathrm{cB}$ \\
\hline & $\mathrm{AA}$ & $17,23 \pm 0,40 \mathrm{bcA}$ & $16,57 \pm 0,47 \mathrm{aAB}$ & $16,63 \pm 1,27^{\mathrm{aA}}$ & $14,97 \pm 0,31 \mathrm{bcB}$ \\
\hline & IS & $19,40 \pm 0,36^{\mathrm{aA}}$ & $15,50 \pm 1,11^{\mathrm{aB}}$ & $16,87 \pm 0,15^{\mathrm{aB}}$ & $16,03 \pm 0,29$ abcB \\
\hline & $\mathrm{AC}$ & $18,57 \pm 1,21 \mathrm{abA}$ & $15,40 \pm 0,3 \mathrm{aB}$ & $16,77 \pm 0,32 \mathrm{aB}$ & $16,23 \pm 0,15 \mathrm{abB}$ \\
\hline
\end{tabular}

As médias seguidas pelas mesmas letras minúsculas na coluna e maiúsculas na linha não diferem entre si pelo teste de Tukey ao nível de $5 \%$ de significância. Controle = água destilada; $\mathrm{AA}=$ ácido ascórbico a $1 \%(\mathrm{~m} / \mathrm{v}) ; \mathrm{IS}=$ isoascorbato de sódio a $1 \%(\mathrm{~m} / \mathrm{v}) ; \mathrm{AC}=$ ácido cítrico a $1 \%(\mathrm{~m} / \mathrm{v})$.

O tratamento por UV-C tem como principal função a sanitização dos frutos, desinfestando-os de patógenos causadores de deteriorações (Sanches et al., 2017). Em tese, não interfere diretamente na acidez do fruto, porém, de forma indireta, pode reduzir alterações na acidez titulável, ao eliminar microrganismos responsáveis por deteriorações em características físico-químicas, entre elas a acidez titulável (Sanches et al., 2017; Abdipour et al., 2019). Acredita-se que não houve incidência alta de patógenos nos tratamentos sem UV-C, por isso tal tratamento apresentou diferenças sutis em relação ao tratamento com UV-C na variável acidez, tendo havido redução na acidez titulável durante o armazenamento devido ao amadurecimento natural do fruto.

Heiffig et al. (2006) observaram redução da acidez titulável ao longo do período de armazenamento de frutos de kiwi cortados em rodelas. Tais autores associam que a redução nos teores de acidez desses frutos esteja relacionada ao dano causado pelo processamento mínimo, o qual aumenta a atividade do sistema de reparo celular, ocasionando mais exigência de consumo de energia metabólica, sendo esta proveniente do metabolismo de açúcares, ácidos e proteínas no processo respiratório (Heiffig et al., 2006). Outra possibilidade é que esse ácido esteja sendo consumido mais rapidamente em reações antioxidativas nos dias subsequentes aos cortes.

Entre os aditivos utilizados nos kiwis minimamente processados com a aplicação de UV-C, o AC apresentou frutos com mais firmeza, não diferindo do IS (Tabela 3). No tratamento sem UV-C, IS manteve mais firmeza da polpa quando comparado ao controle. De modo geral, pode-se observar que o controle, independentemente da aplicação ou não de UV-C, demonstra menos firmeza da polpa dos kiwis. Em maçãs minimamente processadas, nenhuma diferença foi observada em consequência do tratamento com UV-C, AC e da combinação AC + UV-C em comparação com o controle (Chen et al., 2016). 
Tabela 3. Firmeza de polpa de frutos de kiwi minimamente processados em função da utilização da radiação UV-C.

\begin{tabular}{ccc}
\hline & Firmeza & \\
\hline & Com UV-C & Sem UV-C \\
\hline Controle & $5,31 \pm 1,74^{\mathrm{cA}}$ & $6,35 \pm 3,26^{\mathrm{bA}}$ \\
\hline AA & $5,90 \pm 1,66^{\mathrm{bcB}}$ & $7,58 \pm 2,31^{\mathrm{abA}}$ \\
\hline IS & $7,12 \pm 1,04^{\mathrm{abA}}$ & $7,76 \pm 2,51^{\mathrm{aA}}$ \\
\hline AC & $8,10 \pm 1,76^{\mathrm{aA}}$ & $7,16 \pm 1,74^{\mathrm{abA}}$ \\
\hline
\end{tabular}

As médias seguidas pelas mesmas letras minúsculas na coluna e maiúsculas na linha não diferem entre si pelo teste de Tukey ao nível de $5 \%$ de significância. Controle = água destilada; $\mathrm{AA}=$ ácido ascórbico a $1 \%(\mathrm{~m} / \mathrm{v}) ; \mathrm{IS}=$ isoascorbato de sódio a $1 \%(\mathrm{~m} / \mathrm{v}) ; \mathrm{AC}=$ ácido cítrico a 1\% (m/v).

$\mathrm{O} \mathrm{pH}$ dos frutos diminuiu significativamente ao final do período de armazenamento, porém não diferiu entre o uso ou não de UV-C (Tabela 4). Heiffig et al. (2006) não observaram variação dos valores de $\mathrm{pH}$ em kiwis minimamente processados ao longo de 12 dias de armazenamento. Durante o amadurecimento, ácidos orgânicos são usados como substrato no metabolismo da respiração, portanto AT diminui e pH e SS aumentam (Mohsen et al., 2017). Nesse trabalho, observou-se o contrário, ou seja, diminuição do pH. Tal fato pode ser explicado pela baixa diminuição da acidez titulável ao longo do armazenamento (Tabela 2) e pela ausência de resposta do teor de sólidos dos frutos tratados com aditivos (Tabela 5). Deste modo, pode-se inferir que houve baixo metabolismo respiratório, ocasionando reduzida conversão dos ácidos orgânicos em açúcares.

$\mathrm{O}$ teor de fenóis totais nos frutos diminuiu significativamente ao longo do armazenamento, independentemente do uso ou não de UV-C (Tabela 4). Barreto et al. (2016) também observaram redução dos teores de fenóis totais em maçãs minimamente processadas durante o armazenamento refrigerado por 12 dias. A diminuição dos fenóis ao longo do armazenamento se deve à oxidação desses compostos, principalmente pela ação da PPO causada pelo aumento da área de contado do fruto com o $\mathrm{O}_{2}$ em função dos cortes.

O atributo da coloração dos frutos, representado pelo ângulo Hue, reduziu ao longo do armazenamento, independentemente do uso de UV-C (Tabela 4). Essa redução dos valores de ângulo Hue indica amarelecimento da polpa dos frutos. No entanto, Heiffig et al. (2006) não observaram variação do ângulo Hue ao longo do armazenamento de kiwis minimamente processados.

A firmeza dos frutos apresentou diferença entre a utilização e a ausência de UV-C somente no dia 0 , em que kiwis minimamente processados associados a UV-C obtiveram maiores médias dessa variável (Tabela 4). Do dia 3 ao 9 de armazenamento refrigerado dos kiwis minimamente processados, não houve diferença entre a aplicação ou não de UV-C. Portanto, para o armazenamento de kiwis minimamente processados por

Tabela 4. pH, teor de fenóis totais, ângulo Hue e firmeza de kiwis minimamente processados com 0, 3, 6 e 9 dias de armazenamento, em função da utilização da radiação UV-C.

\begin{tabular}{|c|c|c|c|c|}
\hline & Dia 0 & Dia 3 & Dia 6 & Dia 9 \\
\hline \multicolumn{5}{|c|}{$\mathrm{pH}$} \\
\hline Com UV-C & $3,08 \pm 0,09^{\mathrm{bA}}$ & $3,05 \pm 0,12^{\mathrm{aA}}$ & $2,90 \pm 0,08^{\mathrm{bB}}$ & $2,72 \pm 0,04^{\mathrm{aC}}$ \\
\hline Sem UV-C & $3,15 \pm 0,06^{\mathrm{aA}}$ & $3,01 \pm 0,03$ bB & $2,98 \pm 0,05 \mathrm{aB}$ & $2,71 \pm 0,05^{\mathrm{aC}}$ \\
\hline \multicolumn{5}{|c|}{ Fenóis totais } \\
\hline Com UV-C & $118,48 \pm 13,37$ aA & $92,64 \pm 10,88^{\mathrm{aB}}$ & $84,94 \pm 5,80 \mathrm{aBC}$ & $78,99 \pm 8,91 \mathrm{aC}$ \\
\hline Sem UV-C & $121,96 \pm 11,16^{\mathrm{aA}}$ & $83,98 \pm 9,07^{\mathrm{bBC}}$ & $91,73 \pm 13,92 \mathrm{aB}$ & $81,31 \pm 7,44^{\mathrm{aC}}$ \\
\hline \multicolumn{5}{|c|}{ Ângulo Hue } \\
\hline Com UV-C & $113,58 \pm 0,64$ aA & $110,09 \pm 1,65^{\mathrm{aC}}$ & $113,17 \pm 0,74 \mathrm{bAB}$ & $112,22 \pm 0,81^{\mathrm{aB}}$ \\
\hline Sem UV-C & $114,00 \pm 0,57$ aA & $108,93 \pm 1,73 \mathrm{bC}$ & $114,14 \pm 0,96^{\mathrm{aA}}$ & $112,28 \pm 0,90^{\mathrm{aB}}$ \\
\hline \multicolumn{5}{|c|}{ Firmeza } \\
\hline Com UV-C & $8,10 \pm 1,57 \mathrm{bA}$ & $6,54 \pm 1,97 \mathrm{aB}$ & $5,30 \pm 1,68^{a B}$ & $6,49 \pm 1,24 \mathrm{aB}$ \\
\hline Sem UV-C & $10,61 \pm 1,644^{\mathrm{aA}}$ & $6,14 \pm 1,41 \mathrm{aB}$ & $6,01 \pm 1,49 \mathrm{aB}$ & $6,10 \pm 1,71 \mathrm{aB}$ \\
\hline
\end{tabular}

As médias seguidas pelas mesmas letras minúsculas na coluna e maiúsculas na linha não diferem entre si pelo teste de Tukey ao nível de $5 \%$ de significância. 
Tabela 5. Sólidos solúveis totais, pH e firmeza de kiwi minimamente processado com 0, 3, 6 e 9 dias de armazenamento, em função do tratamento com aditivo.

\begin{tabular}{|c|c|c|c|c|}
\hline & Dia 0 & Dia 3 & Dia 6 & Dia 9 \\
\hline \multicolumn{5}{|c|}{ Sólidos solúveis totais } \\
\hline Controle & $12,72 \pm 0,84 \mathrm{aB}$ & $15,08 \pm 1,24 \mathrm{aA}^{\mathrm{aA}}$ & $15,32 \pm 0,55 \mathrm{aA}$ & $14,08 \pm 0,85 \mathrm{aA}$ \\
\hline $\mathrm{AA}$ & $13,02 \pm 0,48^{\mathrm{aA}}$ & $13,27 \pm 0,8^{\mathrm{bA}}$ & $13,50 \pm 0,57^{\mathrm{bA}}$ & $12,45 \pm 0,89 \mathrm{bA}$ \\
\hline IS & $13,43 \pm 1,27$ aA & $13,33 \pm 0,65^{b A}$ & $13,82 \pm 0,88^{b A}$ & $13,55 \pm 0,48^{\mathrm{abA}}$ \\
\hline $\mathrm{AC}$ & $13,28 \pm 0,75^{\mathrm{aA}}$ & $14,22 \pm 1,35$ abA & $14,48 \pm 0,92 \mathrm{abA}$ & $13,57 \pm 0,68^{a b A}$ \\
\hline \multicolumn{5}{|c|}{ pH } \\
\hline Controle & $3,04 \pm 0,09^{\mathrm{bA}}$ & $3,03 \pm 0,07 \mathrm{abA}$ & $2,90 \pm 0,11^{\mathrm{bB}}$ & $2,73 \pm 0,04 \mathrm{aC}$ \\
\hline $\mathrm{AA}$ & $3,13 \pm 0,05$ aA & $3,11 \pm 0,11^{\mathrm{aA}}$ & $2,93 \pm 0,06^{\mathrm{abB}}$ & $2,69 \pm 0,03 \mathrm{aC}$ \\
\hline IS & ${ }^{3}, 16 \pm 0,07$ aA & $3,01 \pm 0,07 \mathrm{bB}$ & $2,93 \pm 0,03 \mathrm{abB}$ & $2,68 \pm 0,04 \mathrm{aC}$ \\
\hline $\mathrm{AC}$ & $3,12 \pm 0,07 \mathrm{abA}$ & $2,97 \pm 0,02^{\mathrm{bB}}$ & $2,99 \pm 0,07 \mathrm{aB}$ & $2,72 \pm 0,05 \mathrm{aC}$ \\
\hline \multicolumn{5}{|c|}{ Firmeza } \\
\hline Controle & $9,51 \pm 2,59$ aA & $5,09 \pm 1,00^{\mathrm{bB}}$ & $4,14 \pm 0,84^{\mathrm{bB}}$ & $4,60 \pm 0,86^{\mathrm{bB}}$ \\
\hline $\mathrm{AA}$ & $9,18 \pm 2,34{ }^{\mathrm{aA}}$ & $5,31 \pm 0,44^{\mathrm{bB}}$ & $5,84 \pm 1,96^{\mathrm{abB}}$ & $6,64 \pm 1,07 \mathrm{aB}$ \\
\hline IS & $9,38 \pm 2,36$ aA & $6,97 \pm 1,19$ abB & $6,08 \pm 1,11$ abB & $7,31 \pm 1,24 \mathrm{aB}$ \\
\hline $\mathrm{AC}$ & $9,33 \pm 1,05$ aA & $7,98 \pm 1,90 \mathrm{aAB}$ & $6,57 \pm 1,38^{a B}$ & $6,63 \pm 1,27 \mathrm{aB}$ \\
\hline
\end{tabular}

As médias seguidas pelas mesmas letras minúsculas na coluna e maiúsculas na linha não diferem entre si pelo teste de Tukey ao nível de $5 \%$ de significância. Controle = água destilada; AA = ácido ascórbico a $1 \%(\mathrm{~m} / \mathrm{v}) ; \mathrm{IS}=$ isoascorbato de sódio a $1 \%(\mathrm{~m} / \mathrm{v}) ; \mathrm{AC}=$ ácido cítrico a $1 \%(\mathrm{~m} / \mathrm{v})$.

mais de três dias, o uso de UV-C ou não é indiferente. Outros estudos corroboram com o fato de a radiação UV-C não afetar a firmeza dos produtos ao longo do armazenamento, como o caso de maçãs minimamente processadas (Manzocco et al., 2011; Chen et al., 2016).

Para o teor de sólidos solúveis, é possível observar que o controle foi o único tratamento que apresentou aumento ao longo dos períodos de armazenamento (Tabela 5), indicando mais maturação dos frutos. De acordo com Jie et al. (2013), o aumento da concentração de açúcar no fruto ocorre com a evolução de maturação de frutos. Aos nove dias de armazenamento, observa-se que o controle apresentou mais teor de sólidos solúveis, porém não diferindo estatisticamente dos tratamentos com aplicação de IS e AC.

$\mathrm{O} \mathrm{pH}$ dos frutos diminuiu em todos os tratamentos durante os períodos de armazenamento (Tabela 5). Aos nove dias de armazenamento, não se observou diferença entre os valores de $\mathrm{pH}$ dos kiwis em função dos aditivos utilizados.

A firmeza dos frutos reduziu ao longo dos períodos de armazenamento, em comparação com a firmeza no início do armazenamento, independentemente do uso dos aditivos (Tabela 5). Aos nove dias de armazenamento, os resultados comprovam a eficiência dos aditivos em manter a integridade dos frutos, em que se observa que os frutos do controle obtiveram perda de firmeza superior à dos demais tratamentos. Em frutos minimamente processados, são encontrados altos valores de redução da firmeza, principalmente pelo fato de os frutos serem cortados e haver alto grau de injuria aos tecidos celulares. Além disso, Rocculi et al. (2005) atribuem a diminuição da firmeza em kiwis minimamente processados a uma série de eventos fisiológicos que incluem degradação enzimática da hemicelulose, solubilização de poliuronídeos, liberação de galactose de polímeros pécticos, inchaço da parede celular e diminuição do teor de água e do potencial osmótico.

Observou-se que os frutos tratados com IS e AC apresentaram maiores valores de luminosidade, ou seja, frutos mais brilhosos em relação aos controles (Tabela 6). Isso demonstra que IS e AC foram eficientes em diminuir o escurecimento dos frutos, em razão do potencial antioxidante desses compostos. Conforme Holzwarth et al. (2013), o AC pode inibir a atividade da PPO. Barreto et al. (2016) também observaram efeito do AA, IS e AC na redução do escurecimento de maçãs 'Fuji suprema' minimamente processadas. Entre os períodos de armazenamento, as luminosidades da polpa dos kiwis diminuíram em relação ao dia 0 (Tabela 7), como consequência das alterações da coloração dos tecidos, principalmente escurecimento. 
Tabela 6. Luminosidade e atividade antioxidante dos frutos de kiwi minimamente processados, tratados com diferentes aditivos.

\begin{tabular}{ccc}
\hline Aditivos & Luminosidade & Atividade antioxidante \\
\hline Controle & $48,70 \pm 4,36^{\mathrm{b}}$ & $723,82 \pm 196,25^{\mathrm{ab}}$ \\
\hline AA & $50,12 \pm 5,27^{\mathrm{ab}}$ & $645,52 \pm 142,70^{\mathrm{b}}$ \\
\hline IS & $50,66 \pm 4,70^{\mathrm{a}}$ & $757,87 \pm 157,65^{\mathrm{a}}$ \\
\hline AC & $51,27 \pm 4,50^{\mathrm{a}}$ & $645,41 \pm 162,50^{\mathrm{b}}$ \\
\hline
\end{tabular}

As médias seguidas pelas mesmas letras minúsculas na coluna não diferem entre si pelo teste de Tukey ao nível de $5 \%$ de significância. Controle $=$ água destilada; AA = ácido ascórbico a 1\% (m/v); IS = isoascorbato de sódio a 1\% (m/v); AC = ácido cítrico a 1\% (m/v).

O teor de atividade antioxidante nos kiwis minimamente processados foi maior com o uso de IS em relação a AA e AC (Tabela 6). Entre os períodos de armazenamento, os dias 3 e 9 apresentaram menores concentrações de atividade antioxidante na polpa dos frutos (Tabela 7). Na literatura, é reportado o efeito dos cortes de tecidos na atividade antioxidante. Os resultados geralmente indicam mudanças na atividade antioxidante como consequência de ferimentos (Gil et al., 2006; Reyes et al., 2007; Heredia \& Cisneros-Zevallos, 2009).

Nesse estudo, a radiação UV-C não alterou o teor da atividade antioxidante nos kiwis minimamente processados (Tabela 1). Porém, em maçãs minimamente processadas, Barreto et al. (2016) observaram que a aplicação de UV-C interferiu na concentração da atividade antioxidante e, após 12 dias de armazenamento refrigerado, essa variável foi maior com o uso de radiação UV-C.

A cromaticidade dos frutos apresentou diferença significativa apenas entre os períodos de armazenamento, tendo se observado diminuição desse atributo ao longo do tempo (Tabela 7). A cromaticidade representa um atributo quantitativo da coloração: quanto maior o croma, maior a saturação das cores perceptíveis aos humanos. Cores neutras possuem baixa saturação, enquanto cores puras possuem alta saturação, portanto são mais brilhantes na percepção humana (Pathare et al., 2013). Deste modo, com o decorrer do tempo de armazenamento, os frutos apresentaram diminuição do brilho. Esse atributo apresentou relação direta com a luminosidade dos frutos. Rocculi et al. (2005) observaram correlação positiva entre a cromaticidade e a luminosidade em frutos de kiwi.

Tabela 7. Luminosidade, cromaticidade e atividade antioxidante de frutos de kiwi minimamente processados tratados com radiação e aditivos e, depois, armazenados em ambiente refrigerado por diferentes períodos.

\begin{tabular}{cccc}
\hline Períodos & Luminosidade & Croma & Atividade antioxidante \\
\hline Dia 0 & $55,44 \pm 2,36^{\mathrm{a}}$ & $34,55 \pm 2,29^{\mathrm{a}}$ & $764,53 \pm 172,37^{\mathrm{a}}$ \\
\hline Dia 3 & $44,91 \pm 2,13^{\mathrm{d}}$ & $21,98 \pm 2,16^{\mathrm{c}}$ & $609,91 \pm 116,33^{\mathrm{b}}$ \\
\hline Dia 6 & $48,75 \pm 3,67^{\mathrm{c}}$ & $27,65 \pm 3,75^{\mathrm{b}}$ & $826,88 \pm 108,91^{\mathrm{a}}$ \\
\hline Dia 9 & $51,64 \pm 2,66^{\mathrm{b}}$ & $28,99 \pm 2,67^{\mathrm{b}}$ & $571,30 \pm 135,76^{\mathrm{b}}$ \\
\hline
\end{tabular}

As médias seguidas pelas mesmas letras minúsculas na coluna não diferem entre si pelo teste de Tukey ao nível de $5 \%$ de significância.

\section{Conclusões}

O uso de aditivos melhora a conservação de frutos de kiwi minimamente processado, com destaque para o isoascorbato de sódio a $1 \%$ e o ácido cítrico a $1 \%$.

Os frutos de kiwi da cultivar 'Bruno' podem ser armazenados por até seis dias sob refrigeração e com o uso de aditivos. Após esse período, a perda de massa é elevada.

O uso de radiação UV-C não alterou a qualidade pós-colheita dos kiwis minimamente processados.

\section{Agradecimentos}

À Coordenação de Aperfeiçoamento de Pessoal de Nível Superior (CAPES) e à Universidade Federal de Pelotas (UFPEL). 


\section{Referências}

Abdipour, M., Hosseinifarahi, M., \& Naseri, N. (2019). Combination method of UV-B and UV-C prevents post-harvest decay and improves organoleptic quality of peach fruit. Scientia Horticulturae, 256, 108564. http://dx.doi.org/10.1016/j.scienta.2019.108564

Agar, I. T., Massantini, R., Hess-Pierce, B., \& Kader, A. A. (1999). Postharvest $\mathrm{CO}_{2}$ and ethylene production and quality maintenance of fresh-cut kiwifruit slices. Journal of Food Science, 64(3), 433-440. http://dx.doi.org/10.1111/j.13652621.1999.tb15058.x

Ambuko, J., Kemunto, N., Hutchinson, M., \& Owino, W. (2017). Comparison of the postharvest characteristics of mango fruits produced under contrasting agro-ecological conditions and harvested at different maturity stages. The Journal of Agricultural Science, 9(8), 181-192. http://dx.doi.org/10.5539/jas.v9n8p181

Barreto, C. F., Kirinus, M. B. M., Silva, P. S., Farias, R. M., Malgarim, M. B., \& Martins, C. R. (2016). Use of additives in combination with UV-C for the conservation of minimally processed 'Fuji Suprema' apples. African Journal of Agricultural Research, 11(48), 4917-4923. http://dx.doi.org/10.5897/AJAR2016.11574

Blum, J., \& Ayub, R. A. (2009). Controle do amadurecimento do kiwi cv. Monty com 1-metilciclopropeno. Revista Brasileira de Fruticultura, 31(1), 39-43. http://dx.doi.org/10.1590/S0100-29452009000100007

Brand-Williams, W., Cuvelier, M. E., \& Berset, C. (1995). Use of a free radical method to evaluate antioxidant activity. Food Science and Technology, 28(1), 25-30.

Carvalho, A. V., \& Lima, L. C. O. (2002). Qualidade de kiwis minimamente processados e submetidos a tratamento com ácido ascórbico, ácido cítrico e cloreto de cálcio. Pesquisa Agropecuária Brasileira, 37(5), 679-685. http://dx.doi.org/10.1590/S0100204X2002000500013

Chakraborty, N., Chakraborty, R., \& Saha, A. K. (2020). Fortified and freeze-dried kiwi fruit (Actinidia deliciosa): Quality and sensory assessment. Brazilian Journal of Food Technology, 23, e2019077. http://dx.doi.org/10.1590/1981-6723.07719

Chen, C., Hu, W., He, Y., Jiang, A., \& Zhang, R. (2016). Effect of citric acid combined with UV-C on the quality of fresh-cut apples. Postharvest Biology and Technology, 111, 126-131. http://dx.doi.org/10.1016/j.postharvbio.2015.08.005

Chin, S. K., Siew, E. S., \& Soon, W. L. (2015). Drying characteristics and quality evaluation of kiwi slices under hot air natural convective drying method. International Food Research Journal, 22(6), 2188-2195.

Fan, X., Huang, R., \& Chen, H. (2017). Application of ultraviolet $C$ technology for surface decontamination of fresh produce. Trends in Food Science \& Technology, 70, 9-19. http://dx.doi.org/10.1016/j.tifs.2017.10.004

Food and Agriculture Organization - FAO. (2018). FAOSTAT: Statistics database. Rome. Retrieved in 2019, June 17, from http://www.fao.org/faostat/en/\#data/TP

Gayán, E., Condón, S., \& Álvarez, I. (2014). Biological aspects in food preservation by ultraviolet light: A review. Food and Bioprocess Technology, 7(1), 1-20. http://dx.doi.org/10.1007/s11947-013-1168-7

Gil, M. I., Aguayo, E., \& Kader, A. A. (2006). Quality changes and nutrient retention in fresh cut versus whole fruits during storage. Journal of Agricultural and Food Chemistry, 54(12), 4284-4296. PMid:16756358. http://dx.doi.org/10.1021/jf060303y

Giovenzana, V., Beghi, R., Civelli, R., \& Guidetti, R. (2015). Optical techniques for rapid quality monitoring along minimally processed fruit and vegetable chain. Trends in Food Science \& Technology, 46(2), 331-338. http://dx.doi.org/10.1016/j.tifs.2015.10.006

Heiffig, L. S., Aguila, J. S., \& Kluge, R. A. (2006). Caracterização físico-química e sensorial de frutos de kiwi minimamente processado armazenados sob refrigeração. Revista Iberoamericana de Tecnología Postcosecha, 8(1), 26-32.

Heredia, J. B., \& Cisneros-Zevallos, L. (2009). The effect of exogenous ethylene and methyl jasmonate on PAL activity: Phenolic profiles and antioxidant capacity of carrots (Daucus carota) under different wounding intensities. Postharvest Biology and Technology, 51(2), 242-249. http://dx.doi.org/10.1016/j.postharvbio.2008.07.001

Holzwarth, M. W., Julia, C. R., \& Kammerer, D. R. (2013). Kammerer Influence of putative polyphenoloxidase (PPO) inhibitors on strawberry (Fragaria $x$ ananassa Duch.) PPO, anthocyanin and color stability of stored purees. Food Science and Technology, 52(2), 116-122.

Instituto Adolfo Lutz - IAL. (2008). Métodos químicos e físicos para análise de alimentos (4. ed., 1020 p.). São Paulo: IAL.

Jie, D., Xie, L., Fu, X., Rao, X., \& Ying, Y. (2013). Variable selection for partial least squares analysis of soluble solids content in watermelon using near-infrared diffuse transmission technique. Journal of Food Process Engineering, 118(4), 387-392. http://dx.doi.org/10.1016/j.jfoodeng.2013.04.027

Kluge, R. A., Geerdink, G. M., Tezotto-Uliana, J. V., Guassi, S. A. D., Zorzeto, T. Q., Sasaki, F. F. C., \& Mello, S. D. C. (2014). Quality of minimally processed yellow bell pepper treated with antioxidants. Semina: Ciências Agrárias, 35(2), 801-812. http://dx.doi.org/10.5433/1679-0359.2014v35n2p801

Maharaj, R. (2015). Effects of abiotic stress (UV-C) induced activation of phytochemicals on the postharvest quality of horticultural crops. In V. A. Rao \& L. G. Rao (Eds.), Phytochemicals: Isolation, characterization and role in human health (Chap. 9, pp. 221-244). London: InTechOpen. http://dx.doi.org/10.5772/60050.

Maldonade, I. R., Ginani, V. C., Riquette, R. F. R., Gurgel-Gonçalves, R., Mendes, V. S., \& Machado, E. R. (2019). Good manufacturing practices of minimally processed vegetables reduce contamination with pathogenic microorganisms. Revista do Instituto de Medicina Tropical de São Paulo, 61, e14. PMid:30785568. http://dx.doi.org/10.1590/s1678-9946201961014

Manolopoulou, E., \& Varzakas, T. (2011). Effect of storage conditions on the sensory quality, colour and texture of fresh-cut minimally processed cabbage with the addition of ascorbic acid, citric acid and calcium chloride. Food and Nutrition Sciences, 2(9), 956-963. http://dx.doi.org/10.4236/fns.2011.29130 
Manzocco, L., Da Pieve, S., Bertolini, A., Bartolomeoli, I., Maifreni, M., Vianello, A., \& Nicoli, M. C. (2011). Surface decontamination of fresh-cut apple by UV-C light exposure: Effects on structure, colour and sensory properties. Postharvest Biology and Technology, 61(2-3), 165-171. http://dx.doi.org/10.1016/j.postharvbio.2011.03.003

Marques, K. M., Mattiuz, B. H., Morgado, C. M. A., Galati, V. C., \& Miguel, A. C. A. (2011). Formas de preparo de figo-da-índia minimamente processado. Revista Brasileira de Fruticultura, 33(spe1), 606-611. http://dx.doi.org/10.1590/S010029452011000500083

Martins, R. N., Mattiuz, B., Santos, L. O., Morgado, C. M. A., \& Mattiuz, C. F. M. (2011). Preservation of minimally processed 'Aurora-1' peaches using additives. Revista Brasileira de Fruticultura, 33(4), 1229-1239. http://dx.doi.org/10.1590/S010029452011000400023

Mendoza-Goméz, V., Calderón-Santoyo, M., Bautista-Rosales, P. U., Ortiz-Basurto, R. I., Jimenez-Sánchez, D. E., \& RagazzoSánchez, J. A. (2017). Antibrowning agents added in polymeric coating applied to avocado fruit slices. Interciencia, 42(12), 812 817.

Mohsen, R., Elham, F., Hamidreza, A., \& Sedigheh, A. (2017). Effect of gelatin-based edible coatings incorporated with Aloe vera and black and green tea extracts on the shelf life of fresh-cut oranges. Journal of Food Quality, 2017, 9764650.

Moreno, M. B., Cantillano, R. F. F., Rombaldi, C. V., \& Manica-Berto, R. (2016). Qualidade de maçã 'Fuji' minimamente processada sob armazenamento refrigerado e tratamento com aditivos. Revista Brasileira de Fruticultura, 38(4), e-532.

Ochoa-Velasco, C. E., \& Guerrero-Beltrán, J. Á. (2012). Ultraviolet-C light effect on pitaya (Stenocereus griseus) juice. Journal of Food Research, 1(2), 60-70. http://dx.doi.org/10.5539/jfr.v1n2p60

Pal, R. S., Kumar, V. A., Arora, S., Sharma, A. K., Kumar, V., \& Agrawal, S. (2015). Physicochemical and antioxidant properties of kiwifruit as a function of cultivar and fruit harvested month. Brazilian Archives of Biology and Technology, 58(2), $262-271$. http://dx.doi.org/10.1590/s1516-8913201500371

Pathare, P. B., Opara, U. L., \& Al-Said, F. A. (2013). Colour measurement and analysis in fresh and processed foods: A review. Food and Bioprocess Technology, 6(1), 36-60. http://dx.doi.org/10.1007/s11947-012-0867-9

Pegoraro, C., Storch, T. T., Crizel, G. R., Ferreira, W. A., \& Girardi, C. L. (2016). Atmosfera controlada associada ao 1metilciclopropeno na preservação da qualidade de kiwi 'tewi'. Brazilian Journal of Food Technology, 19(0), e2014078. http://dx.doi.org/10.1590/1981-6723.7814

Reyes, L. F., Emilio Villarreal, J., \& Cisneros-Zevallos, L. (2007). The increase in antioxidant capacity after wounding depends on the type of fruit or vegetable tissue. Journal of Agricultural and Food Chemistry, 101(3), 1254-1262. http://dx.doi.org/10.1016/j.foodchem.2006.03.032

Rocculi, P., Romani, S., \& Dalla Rosa, M. (2005). Effect of MAP with argon and nitrous oxide on quality maintenance of minimally processed kiwifruit. Postharvest Biology and Technology, 35(3), 319-328. http://dx.doi.org/10.1016/j.postharvbio.2004.09.003

Rodgers, S. (2016). Minimally processed functional foods: Technological and operational pathways. Journal of Food Science, 81(10), R2309-R2319. PMid:27603331. http://dx.doi.org/10.1111/1750-3841.13422

Sanches, A. G., Silva, M. B., Moreira, E. G. S., Cosme, S. S., \& Cordeiro, C. A. M. (2017). "Radiação uv-c na longevidade póscolheita de tangerinas sob refrigeração. Agrarian, 10(36), 129-135. http://dx.doi.org/10.30612/agrarian.v10i36.5372

Singleton, V. L., Orthofer, R., \& Lamuela-Raventós, R. M. (1999). Analysis of total phenols and other oxidation substrates and antioxidants by means of folin-ciocalteu reagent. Methods in Enzymology, 299(4), 152-178. http://dx.doi.org/10.1016/S00766879(99)99017-1

Vieites, R. L., Evangelista, E. M., Campos, A. J., \& Moreira, G. C. (2004). Effect of the packing and the gama irradiation on the microbiological contamination control of the minimally processed mango. Semina: Ciências Agrárias, 25(3), 197-206. http://dx.doi.org/10.5433/1679-0359.2004v25n3p197

Vilas-Boas, E. V. B., Reis, C. M. F., Melo, A. A., \& Martins, B. (2009). Use of chemical mixtures for firmness maintenance of fresh-cut 'Silver' banana. Ciência e Agrotecnologia, 1, 237-244.

Wu, Z. S., Zhang, M., \& Wang, S. (2012). Effects of high pressure argon treatments on the quality of fresh-cut apples at cold storage. Food Control, 23(1), 120-127. http://dx.doi.org/10.1016/j.foodcont.2011.06.021 